\title{
Construction of a novel vector expressing Survivin-shRNA and fusion suicide gene yCDglyTK and its application in inhibiting proliferation and migration of colon cancer cells
}

\author{
LING YE $^{1}$, YUAN YANG ${ }^{1}$, XIN-YU MA ${ }^{1}$, DAN LI ${ }^{1}$, MEI-LI XU ${ }^{1}$, PAN TAN $^{1}$, \\ LI-MIN LONG ${ }^{1}$, HAI-QIN WANG ${ }^{1}$, TING LIU ${ }^{2}$ and YONG-HONG GUO ${ }^{1}$ \\ ${ }^{1}$ Department of Geriatrics, The Second Xiangya Hospital of Central South University, Changsha, Hunan 410011; \\ ${ }^{2}$ Department of Gastroenterology, Xiangya Hospital of Central South University, Changsha, Hunan 410008, P.R. China
}

Received March 2, 2016; Accepted June 2, 2017

DOI: $10.3892 / e t m .2017 .5154$

\begin{abstract}
Despite progress achieved in cancer chemotherapy in recent decades, adverse effects remain a limiting factor for a number of patients with colorectal cancer, suggesting the requirement for novel therapeutic strategies. Gene therapy appears to be a promising strategy for treating cancer. The present study aimed to investigate the anti-tumor effect of a combined gene therapy, using Survivin downregulation by RNAi and a fusion suicide gene yCDglyTK therapy system. A triple-gene vector expressing Survivin-targeted small hairpin RNA (Survivin-shRNA) and fusion suicide gene yCDglyTK was constructed, and administered to HCT116 cells. Survivin expression decreased significantly and yCDglyTK fusion gene expression was confirmed by both reverse transcription-quantitative polymerase chain reaction and western blot analysis. Introduction of Survivin-shRNA into yCDglyTK/prodrug system eradicated colon cancer cells and induced apoptosis more effectively. Furthermore, this therapeutic system is able to inhibit the migration of HCT116 cells. These results indicate that the recombinant plasmid may serve as a novel gene therapy approach to treat colorectal carcinoma.
\end{abstract}

\section{Introduction}

Colorectal cancer (CRC) is the third most common cancer worldwide (1). Of patients with CRC, 50-70\% are diagnosed at advanced stages (2), and adjuvant chemotherapies are recommended in addition to radical surgery to decrease the possibility of recurrence and increase the success rate. However, adjuvant chemotherapies, which are administrated systemically, are

Correspondence to: Dr Yong-Hong Guo, Department of Geriatrics, The Second Xiangya Hospital of Central South University, 139 Renmin Road, Changsha, Hunan 410011, P.R. China E-mail: guoyhong@sina.cn

Key words: Survivin, suicide gene, RNAi, combination gene therapy unable to selectively target cancerous cells and, in turn cause substantial toxicity (3), resulting in an impaired quality of life for patients. Therefore, novel therapeutic strategies are required.

Gene-direct enzyme/prodrug therapy (GEPT), also named suicide gene therapy, has received considerable attention due to its powerful anti-tumor efficacy without side effects $(4,5)$. GEPT is based on the intracellular delivery of genes encoding enzymes that convert nontoxic prodrugs into highly cytotoxic metabolites (6). Well-characterized GEPTs include the herpes simplex virus thymidine kinase/ganciclovir (HSV-TK/GCV) and cytosine deaminase/5-fluorocytosine (CD/5-FC) (7). TK activates GCV to its cytotoxic triphosphate derivative, which inhibits cellular DNA synthesis, whereas CD deaminates 5-FC into the highly toxic 5-fluorouracil (5-FU), which may interfere with nucleoside metabolism and lead to targeted cell death (8). However, GEPT is thought to be insufficient to cure cancer alone (9). Previously, a number of studies have aimed to enhance the therapeutic effect of GEPT through combination with other gene therapies, including immuno-gene (10), anti-oncogene (11) and inhibition of multiple drug resistance gene based on RNAi (12).

Survivin, which is known to be a member of the inhibitor of apoptosis protein family (13), is overexpressed in a number of human cancer types, including CRC (14-16). Recent studies have indicated that Survivin serves an essential role in tumor growth, infiltration and metastasis, and that it is closely associated with the chemo-resistance of cancer cells $(17,18)$. Survivin has become a focus in cancer therapy. RNA interference (RNAi) technology, based on sequence-specific interactions between small interfering RNA (siRNA) and mRNA (19), is post-transcriptional gene silencing. Inhibition of Survivin by RNAi has been demonstrated to restrain tumor growth and metastasis, and increase sensitivity to anti-tumor agents (20). The anti-tumor effect of GEPT is mediated by cytotoxic metabolites of prodrugs, such as 5-FU. The downregulation of Survivin may help maintain the sensitivity of colorectal cancer cells to the cytotoxic drugs. Therefore, a combination of Survivin-targeted RNAi and the suicide gene may exhibit synergistic effects for cancer treatment.

In the present study, a triple-gene vector expressing Survivin-shRNA and fusion suicide gene yCDglyTK was 
constructed to assess the feasibility of a novel therapeutic vector system involving a combination of GEPT with Survivin-targeted RNAi therapy. This novel vector was delivered into HCT116 cells (a colon cancer cell line) by calcium phosphate nanoparticles (CPNPs), and the anti-tumor effect was studied in vitro.

\section{Materials and methods}

Reagents. Restriction enzymes BsaI, MluI, XhoI and NheI were purchased from MBI Fermentas (Thermo Fisher Scientific, Inc., Waltham, MA, USA). T4-DNA ligase (New England Biolabs, Inc., Ipswich, MA, USA), rTaq DNA polymerase (Takara Biotechnology Co., Ltd., Dalian, China), DNA Marker IV, DNA Marker DL2000 (YRbio; Changsha, China), pYr1.1 vector (YRbio) and pUC57 (YRbio) were applied. Lipofectamine 2000 (Invitrogen, Thermo Fisher Scientific, Inc.), MinElute Gel Extration Kit ( Qiagen GmbH; Hilden, Germany), Geneticin (G418; Thermo Fisher Scientific, Inc.), TRIzol reagent (Invitrogen; Thermo Fisher Scientific, Inc.), ReverTra Ace reverse transcription kit (Toyobo Co., Ltd., Osaka, Japan), 2X Taq PCR MasterMix (Tiangen Biotech Co., Ltd., Beijing, China), rabbit anti-Survivin antibody (ab76424; 1;5,000; Abcam, Cambridge, UK), mouse anti-TK antibody (sc-53331; 1:200; Santa Cruz Biotechnology, Inc., Dallas, TX, USA), mouse anti- $\beta$-actin antibody (A5316; $1 ; 5,000$; Sigma-Aldrich; Merck KGaA, Darmstadt, Germany), goat anti-rabbit secondary antibody (SA00001-2; 1:2,000; Proteintech Group, Inc., Chicago, IL, USA), goat anti-mouse secondary antibody (SA00001-1; 1:2,000; Proteintech Group, Inc.), rabbit anti-CD antibody (10348-924; 1:200; VWR International; Randor, PA, USA), FITC-Goat Anti-Rabbit antibody (SA00003-2; 1:100; Proteintech Group, Inc.), GCV (Sigma-Aldrich; Merck KGaA), 5-FC (Sigma-Aldrich; Merck KGaA), MTT solution (Sigma-Aldrich; Merck KGaA), dimethyl sulfoxide (Promega Corporation) and pyridine iodide (PI; Sigma-Aldrich; Merck KGaA) were also used in the study.

Construction of Survivin-shRNA expressing plasmid. The Survivin mRNA sequence in GenBank (https://www. ncbi.nlm.nih.gov/gene) was searched, and three Survivinspecific target sequences were selected according to the RNAi design tool (https://sg.idtdna.com/site/order/designtool/index/DSIRNA_CUSTOM). The first siRNA sequence targeted the coding region 118-138 (5'-GAGGCTGGCTTC ATCCACTGC-3'), the second sequence targeted the coding region 323-342 (5'-GAGCCA AGA ACA AAATTGC-3') and the third sequence targeted the coding region 387-405 (5'-GAAAGTGCGCCGTGCCAT-3'). Oligonucleotides that encoded the corresponding small hairpin RNA (shRNA) were synthesized commercially (Yrbio, Changsha, China), and the sequences are presented in Table I.

The oligonucleotides were annealed in annealing buffer $(10 \mathrm{mM}$ Tris- $\mathrm{HCl} \mathrm{pH} 8.0,50 \mathrm{mM} \mathrm{NaCl}$ and $1 \mathrm{mM}$ EDTA), and pYr1.1 vector was digested with $B s a \mathrm{I}$ at $37^{\circ} \mathrm{C}$ overnight. Subsequently, the linear fragment of pYr1.1 and the annealing products were connected at $4^{\circ} \mathrm{C}$ overnight to construct pYr1.1-Survivin-sh1, pYr1.1-Survivin-sh2 and pYr1.1-Survivin-sh3, respectively. The expression of shRNA was regulated by the U6 promoter. Then the three interfering plasmids were sequenced. The three interfering plasmids were then transfected into HCT116 cells using Lipofectamine 2000 according to the manufacturer's instructions, and the protein expression of Survivin was evaluated by western blot analysis, as described below. pYr1.1-Survivin-sh2 was confirmed to be the most effective interfering plasmid.

Construction of the triple-gene plasmid. The suicide gene should be expressed only in cancer cells, and the human telomerase reverse transcriptase promoter (hTERTp) was used to observe target expression. The hTERTp was synthesized by Yrbio, according to a previous study (21), and the sequence was: 5'-ACGCGTGCTCCCAGTGGATTCGCGGGCACA GACGCCCAGGACCGCGCTCCCCACGTGGCGGAG GGACTGGGGACCCGGGCACCCGTCCTGCCCCTTCAC CTTCCAGCTCCGCCTCCTCCGCGCGGACCCCGCCCC GTCCCGACCCCTCCCGGGTCCCCGGCCCAGCCCCCT CCGGGCCCTCCCAGCCCCTCCCCTTCCTTTCCGCGG CCCCGCCCTCTCCTCGCGGCGCGAGTTTCAGGCAGC GCTGCGTCCTGCTGCGCACGTGGGAAGCCCTGG CCCCGGCCACCCCCGCGGCTAGC-3' (the underlined sections were $M l u \mathrm{I}$ and $\mathrm{Nhe}$ I restriction sites, respectively), and was subcloned into pUC57 vector, which was named pUC57-hTERTp. pUC57-hTERTp and pYr1.1 were digested by $M l u \mathrm{I}$ and $N h e \mathrm{I}$ at $37^{\circ} \mathrm{C}$ overnight, respectively, and the linear fragments were connected by T4 DNA ligase at $4^{\circ} \mathrm{C}$ overnight to construct pYr1.1-hTERTp. A plasmid carrying fusion suicide gene yCDglyTK was constructed as described in our previous study (22), which was stored in the department of Gastroenterology, Xiangya Hospital of Central South University (Changsha, China). The fusion suicide gene yCDglyTK was amplified through polymerase chain reaction (PCR). Primer sequences used were as follows: P1, 5'-CTA GCTAGCGCCACCATGGTGACAGGGGGAATGGCAA-3'

(NheI restriction site was introduced), and $\mathrm{P} 2,5^{\prime}-\mathrm{CCGCTC}$ GAGTCAGTTAGCCTCCCCCATCT-3' (XhoI restriction site was introduced). The reaction mixture for PCR contained the following: $0.25 \mu \mathrm{l}$ P1 $(10 \mu \mathrm{M}), 0.25 \mu \mathrm{l} 2$ (10 $\mu \mathrm{M}), 19.75 \mu \mathrm{l}$ $\mathrm{dH}_{2} \mathrm{O}, 2.5 \mu \mathrm{l}$ 10X LA PCR buffer ( $\mathrm{Mg}^{2+}$ Plus), $1 \mu \mathrm{l} \mathrm{dNTPs}$ $(2.5 \mathrm{mM}), 0.25 \mu \mathrm{l} \mathrm{LA}$ Taq polymerase and $1 \mu \mathrm{l}$ template. The thermal cycle profile for PCR was $94^{\circ} \mathrm{C}$ for $5 \mathrm{~min}$, followed by 30 cycles of $20 \mathrm{sec}$ at $94^{\circ} \mathrm{C}, 25 \mathrm{sec}$ at an annealing temperature of $58^{\circ} \mathrm{C}, 105 \mathrm{sec}$ at $72^{\circ} \mathrm{C}$, and an additional $3 \mathrm{~min}$ incubation at $72^{\circ} \mathrm{C}$ following completion of the last cycle for extension. Following electrophoresis on $1 \%$ agarose gel, PCR products were extracted and stored at $4^{\circ} \mathrm{C}$.

PCR products of yCDglyTK and pYr1.1-hTERTp were subsequently digested by $\mathrm{Nhe} \mathrm{I}$ and $\mathrm{XhoI}$ respectively at $37^{\circ} \mathrm{C}$ overnight, and the two linear fragments were connected at $4^{\circ} \mathrm{C}$ overnight to develop the plasmid pYr1.1-hTERTp-yCDglyTK. In this process, the enhanced green fluorescent protein (EGFP) of pYr1.1-hTERTp was replaced by yCDglyTK.

pUC57-hTERTp and pYr1.1-Survivin-sh2 were digested by $\mathrm{MluI}$ and $\mathrm{NheI}$ respectively at $37^{\circ} \mathrm{C}$ overnight, and the linear fragments were connected at $4^{\circ} \mathrm{C}$ overnight to construct pYr-1.1-hTERTp-Survivin-sh2. Subsequently, PCR products of yCDglyTK and pYr-1.1-hTERTp-Survivin-sh2 were digested by NheI and XhoI respectively at $37^{\circ} \mathrm{C}$ overnight, and the two linear fragments were connected (at $4^{\circ} \mathrm{C}$ overnight) to construct 
Table I. Sequences of oligonucleotides encoding Survivin-shRNA.

Survivin-shRNA

Survivin-sh1

Survivin-sh2

Survivin-sh3
Sequences of oligonucleotides

Forward:5'-CACCGAGGCTGGCTTCATCCACTGCCTCGAGGCAGTGGATGAAGCCAGC CTCTTTTTTG-3'

Reverse:5'-AGCTCAAAAAAGAGGCTGGCTTCATCCACTGCCTCGAGGCAGTGGATGA AGCCAGCCTC-3

Forward: 5'-CACCGAGCCAAGAACAAAATTGCTTCAAGAGAGCAATTTTGTTCTTGG CTCTTTTTTG-3'

Reverse: 5'-AGCTCAAAAAAGAGCCAAGAACAAAATTGCTCTCTTGAAGCAATTTTG TTCTTGGCTC-3'

Forward: 5'-CACCGAAAGTGCGCCGTGCCATCTTCAAGAGAGATGGCACGGCGCACT TTCTTTTTTG-3'

Reverse: 5'-AGCTCAAAAAAGAAAGTGCGCCGTGCCATCTCTCTTGAAGATGGCACG GCGCACTTTC-3'

Table II. Plasmids used in the present study.

\begin{tabular}{llll}
\hline Plasmids & Abbreviations & Promoters & \multicolumn{1}{c}{ Inserts } \\
\hline pYr1.1 & pYr1.1 & hU6 & EGFP \\
pYr1.1-Survivin-sh1/2/3 & shSur1/2/3 & hU6 & Survivin-shRNA1/2/3 \\
pYr1.1-hTERTp & pYr1.1-hTERTp & hTERTp and hU6 & EGFP \\
pYr1.1-hTERTp-yCDglyTK & hTERTp-CDTK & hTERTp and hU6 & yCDglyTK \\
pYr1.1-hTERTp-yCDglyTK-sh Survivin2 & CDTK-shSur & hTERTp and hU6 & yCDglyTK and Survivin-shRNA2
\end{tabular}

EGFP, enhanced green fluorescent protein; hTERTp, the human telomerase reverse transcriptase promoter; CDTK, fusion suicide gene involving yeast CD gene and HSV-TK gene; shSur, Survivin-targeted small hairpin RNA.

a novel triple-gene vector pYr1.1-hTERTp-yCDglyTK-shSurvivin2. Plasmids used in the current study are presented in Table II.

Cell line and cell culture. HCT116 (a human colon cancer cell line) and human fibroblasts obtained from the Central Laboratory of the Second Xiangya Hospital, Central South University (Changsha, China), were used in the present study. The present study was approved by the ethics committee of the Second Xiangya Hospital, Central South University (Changsha, China) and informed consent was obtained from patients prior to the use of human tissue. Cells were cultured in RPMI 1640 medium (Hyclone; GE Healthcare Life Sciences, Logan, UT, USA), supplemented with $10 \%$ fetal bovine serum (FBS, Hyclone; GE Healthcare Life Sciences, Logan, UT, USA) and maintained at $37^{\circ} \mathrm{C}$ in a humidified atmosphere of $5 \% \mathrm{CO}_{2}$ and $95 \%$ air.

Analysis of EGFP expression. HCT116 and human fibroblasts were seeded in 6-well plates at a density of $2 \times 10^{5}$ cells/well. As described in a previous study (22), calcium phosphate nanoparticles (CPNPs) were produced, and $2 \mu \mathrm{g}$ DNA (pYr1.1-hTERTp) was mixed with $20 \mu \mathrm{g}$ CPNPs to form the CPNP-DNA complex, which was then added to each well. The expression of EGFP was analyzed $48 \mathrm{~h}$ later using a fluorescence microscope (DMI 4000B; Leica Microsystems GmbH, Wetzlar, Germany).
Stable transfection in vitro. HCT116 cells were seeded in 6 -well plates at a density of $2 \times 10^{5}$ cells per well. When the cell monolayer reached $70-80 \%$ confluence, hTERTp-CDTK and CDTK-shSur were mixed with CPNPs respectively. Each of the CPNP-DNA complexes was added to different 6-well plates as described previously (22). The next day, a 1:10 passage of the transfected HCT116 cells was performed, followed by the addition of $400 \mu \mathrm{g} / \mathrm{ml} \mathrm{G} 418$ for selection. G418-resistant clones were isolated and expanded in RPMI-1640 culture medium containing $200 \mu \mathrm{g} / \mathrm{ml} \mathrm{G} 418$. Surviving colonies transfected with hTERTp-CDTK or CDTK-shSur were renamed HCT/CDTK, or HCT/CDTK-shSur, respectively, and subjected to further studies.

Reverse transcription-PCR (RT-PCR). Total RNA from parental and transfected HCT116 cells was extracted using TRIzol reagent. The quantity and quality of RNA were assessed by absorbance at $260 \mathrm{~nm}$ and $280 \mathrm{~nm}$ using an ultraviolet spectrophotometer (DU800; Beckman Coulter, Inc., Brea, CA, USA). The RT reaction was performed using the ReverTra Ace reverse transcription kit according to the manufacturer's protocol. Subsequently, PCR was performed on the cDNA product. For yCDglyTK, a PCR product of 707 bp was produced by forward primer 5'-GGGAGATTAGAGGGCAAA GTGT-3' and reverse primer 5'-ACGGCGTCGGTCACGGCA TAA-3'. For Survivin, a PCR product of $107 \mathrm{bp}$ was produced 
by forward primer 5'-CATCCTGCGTCTGGACCTGG-3' and reverse primer 5'-TAATGTCACGCACGATTTCC-3'. $\beta$-actin was used as an internal control, and the forward primer was 5'-AGCGAGCATCCCCCAAAGTT-3' and the reverse primer was 5'-GGGCACGAAGGCTCATCATT-3'. The thermal cycle profile for PCR was $94^{\circ} \mathrm{C}$ for $3 \mathrm{~min}$, followed by 28 cycles of $30 \mathrm{sec}$ at $94^{\circ} \mathrm{C}, 30 \mathrm{sec}$ at an annealing temperature of $55^{\circ} \mathrm{C}$ and $60 \mathrm{sec}$ at $72^{\circ} \mathrm{C}$. PCR products were electrophoresed on $2 \%$ agarose gels, and visualized using gel image analysis system (BIO-PRO, SIM International group Co., Ltd., Los Angeles, CA, USA) and analyzed by Bandscan 5.0 ( http://www.bbioo .com/download/58-140-1.html).

Western blot analysis. Parental and transfected HCT116 cells were lysed in radioimmunoprecipitation assay buffer on ice containing phenylmethylsulfonyl fluoride for $30 \mathrm{~min}$ with occasional agitation. The lysates were transferred to E-tubes and clarified by centrifugation at $14,000 \mathrm{x}$ g for $15 \mathrm{~min}$ at $4^{\circ} \mathrm{C}$. The supernatant was collected and protein concentrations were evaluated using a BCA protein assay. Identical amounts $(40 \mu \mathrm{g}$ protein) of cell lysates were separated via $15 \%$ SDS-PAGE and transferred to polyvinylidene fluoride membranes (GE Healthcare, Chicago, IL, USA). The membranes were incubated in blocking solution, consisting of 5\% skim milk in Tris buffered saline with Tween-20 [10 mM Tris- $\mathrm{HCl}$ (pH 8.0), $150 \mathrm{mM} \mathrm{NaCl}$, and $0.1 \%$ Tween-20], for $1 \mathrm{~h}$ at room temperature, then probed with rabbit anti-Survivin antibody, rabbit anti-TK antibody or mouse anti- $\beta$-actin antibody at $44^{\circ} \mathrm{C}$ overnight. This was followed by incubation with their respective peroxidase-conjugated secondary antibodies for $1.5 \mathrm{~h}$ at room temperature. The blots were visualized by the enhanced chemiluminescence detection system (GE Healthcare) and analyzed by Bandscan 5.0.

Immunofluorescence assay. Parental and transfected HCT116 cells $\left(2 \times 10^{5}\right.$ cells per well) were fixed in $4 \%$ formaldehyde for $20 \mathrm{~min}$ at room temperature. Cells were washed with cold PBS three times, then permeabilized in $0.3 \%$ Triton $\mathrm{X}-100$ and blocked with $1 \%$ bovine serum albumin for $30 \mathrm{~min}$ at room temperature. The cells were then treated with rabbit anti-CD antibody overnight at $4^{\circ} \mathrm{C}$ in a humidified chamber. Then, cells were incubated with anti-rabbit immunoglobulin $\mathrm{G}$-fluorescein isothiocyanate antibody for $1 \mathrm{~h}$ at $37^{\circ} \mathrm{C}$ in the dark. After being washed three times with PBS, coverslips were mounted with a drop of mounting medium (Beyotime Institute of Biotechnology, Shanghai, China), sealed with clear nail polish and visualized using a fluorescence microscope (magnification, x200).

MTT assay. HCT116 cells (transfected and untransfected) were seeded in 96-well plates at a density of 6,000 cells per well. The next day (at $37^{\circ} \mathrm{C}$ ), cells were treated with prodrugs: $200 \mu \mathrm{g} / \mathrm{ml}$ 5-FC and $16 \mu \mathrm{g} / \mathrm{ml} \mathrm{GCV}$, which have been confirmed to have limited toxicity on untransfected cells in a previous study (22), and cell viability was measured after 24, 48, 72 and $96 \mathrm{~h}$ of incubation at $37^{\circ} \mathrm{C}$. A volume of $20 \mu \mathrm{l}$ MTT solution $(5 \mathrm{mg} / \mathrm{ml})$ was added and cells were further incubated at $37^{\circ} \mathrm{C}$ for $4 \mathrm{~h}$. Then, the culture medium was removed and replaced with $200 \mu 1$ dimethyl sulfoxide to dissolve the blue crystals. The optical density (OD) was determined using a multi-well plate reader (Stat-Fax-2100; Awareness Technologies, Westport, CT, USA) by measuring absorbance at $570 \mathrm{~nm}$ (OD570), with the absorbance at $690 \mathrm{~nm}$ as a reference. The background absorbance of medium was also subtracted. Cell growth curves were produced with culture time on the horizontal axis and OD570 on the vertical axis.

Cell apoptosis analyzed by flow cytometry. A flow cytometry assay was performed to evaluate the loss of cell viability in each experimental group. Parental and transfected HCT116 cells were seeded into $75 \mathrm{~cm}^{2}$ cell culture flasks at a density of $2 \times 10^{6}$ cells per flask. RPMI 1640 medium (supplemented with $10 \%$ FBS) with $200 \mu \mathrm{g} / \mathrm{ml} \mathrm{5-FC}$ and $16 \mu \mathrm{g} / \mathrm{ml} \mathrm{GCV}$ was added when the cells reached $70 \%$ confluence. $48 \mathrm{~h}$ later, the cells were pelleted by centrifugation at $800 \mathrm{x} \mathrm{g}$, washed with cold PBS twice, fixed in $75 \%$ ethanol for $30 \mathrm{~min}$ at $4^{\circ} \mathrm{C}$ and resuspended in a staining solution of PI $(50 \mathrm{mg} / \mathrm{ml})$ for $30 \mathrm{~min}$ at $37^{\circ} \mathrm{C}$. Finally, the cell apoptosis rate was analyzed using flow cytometry (FACSCanto, BD Biosciences; San Jose, CA, USA).

Migration assay. A wound healing assay was applied to analyze cell migration. HCT116 cells (transfected and untransfected) were seeded in 6-well plates at a density of $5 \times 10^{5}$ cells/well in RPMI 1640 medium with $10 \%$ FBS for $24 \mathrm{~h}$ at $37^{\circ} \mathrm{C}$ to reach $95 \%$ confluence. The monolayers were then scratched with a $200 \mu$ l pipette tip. The cells were washed three times with PBS, and cultured in RPMI 1640 medium without FBS for $24 \mathrm{~h}$ at $37^{\circ} \mathrm{C}$. Migration of the cells was detected under a light microscope. The wound margin distances between the two edges of the migrating cell sheets were measured at 0 and $24 \mathrm{~h}$ following scratching. The relative migrating distance of cells was measured as follows: Distance of cell migration/the distance measured at $0 \mathrm{~h}$.

Statistical analysis. All results were expressed as mean \pm standard deviation. Statistical analysis was performed using SPSS version 13.0 (SPSS, Inc., Chicago, IL, USA) The Student's t-test and one-way analysis of variance assessments followed by the Student-Newman-Keuls test was performed. $\mathrm{P}<0.05$ was considered to indicate statistically significant differences.

\section{Results}

Construction of the plasmid pYr1.1-hTERTp-yCDglyTKshSurvivin2. Three interfering plasmids targeting Survivin were constructed and the most effective plasmid, pYr1.1-Survivin-sh2, was selected. Subsequently, hTERTp was cloned into pYr1.1 to obtain pYr1.1-hTERTp, and the specificity of hTERTp was confirmed by fluorescence microscopy, as presented in Fig. 1A. Then, yCDglyTK was cloned into pYr1.1-hTERTp to generate pYr1.1-hTERTp-yCDglyTK. Finally, Survivin-shRNA from pYr1.1-Survivin-sh2 was cloned into pYr1.1-hTERTp-yCDglyTK to develop the triple-gene plasmid pYr1.1-hTERTp-yCDglyTK-shSurvivin2. In this novel triple-expressing plasmid, the Survivin-shRNA sequence was driven by a U6 promoter, whereas fusion suicide gene yCDglyTK was regulated by hTERTp. The construction scheme of the triple-gene plasmid 
A
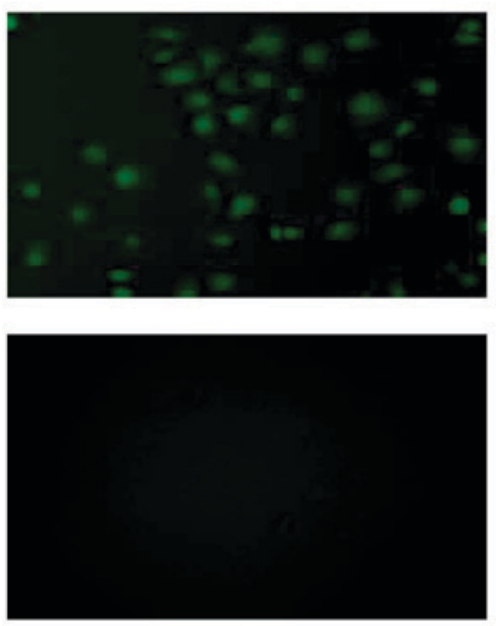

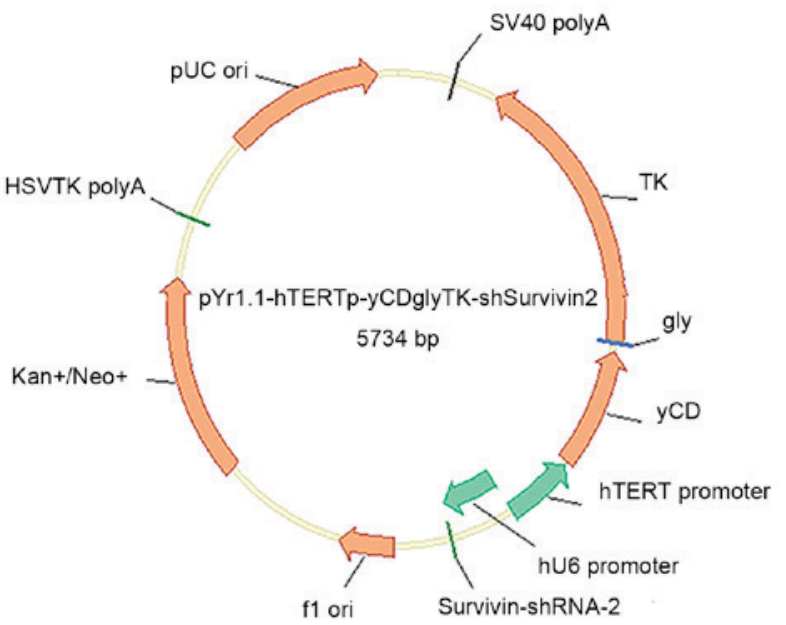

Figure 1. Construction of the pYr1.1-hTERTp-yCDglyTK-shSurvivin2 plasmid. (A) Expression of EGFP in HCT116 cells and human fibroblasts by transfection with pYr1.1-hTERTp. Top image demonstrates the EGFP-positive expression of HCT116 cells by fluorescence microscopy; bottom image demonstrates the negative expression of EGFP in fibroblasts (magnification, x200). (B) Construction scheme of a novel plasmid pYr1.1-hTERTp-yCDglyTK-shSurvivin2. EGFP, enhanced green fluorescent protein; TK, thymidine kinase; yCD, yeast cytosine deaminase; hTERT, human telomerase reverse transcriptase; Survivin-shSur, Survivin-targeted small hairpin RNA.

A
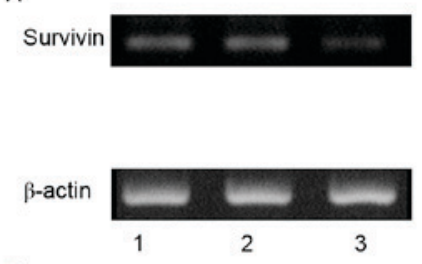

C

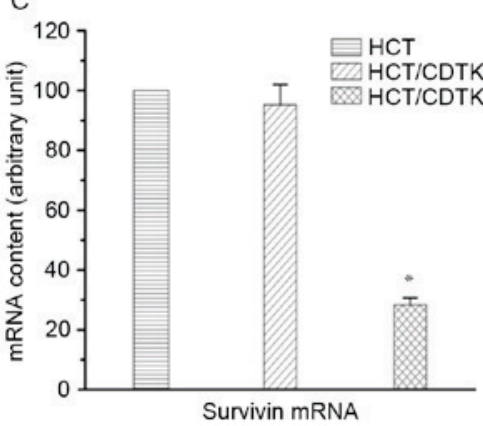

B

Survivin

$\beta$-actin
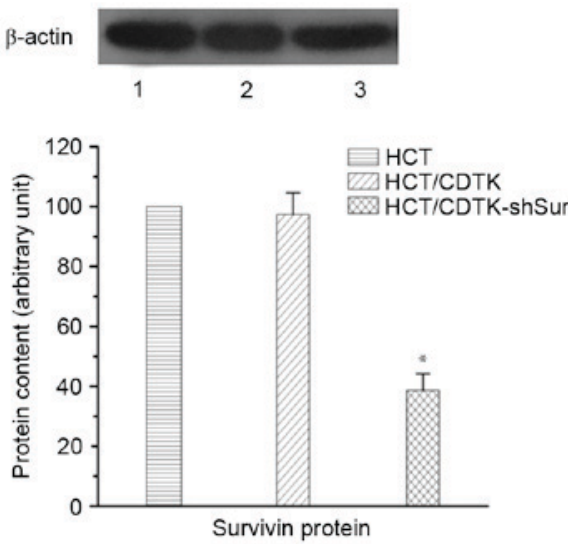

Figure 2. Inhibition of Survivin mRNA and protein expression by transfection with CDTK-shSur. Representative Survivin mRNA and protein expression were analyzed by (A) RT-qPCR and (B) western blot analysis, respectively. $\beta$-actin was used as an internal control. Lane 1, parent HCT116; lane 2, HCT/CDTK; lane 3, HCT/CDTK-shSur. (C) Density of each band was measured, densities of Survivin were normalized against corresponding $\beta$-actin signals, and relative intensities were expressed in arbitrary units where the intensity of parent HCT116 cells was set to $100 \%$. ${ }^{*} \mathrm{P}<0.01 \mathrm{vs}$. all other groups. The data are expressed as mean + standard deviation from three independent experiments. CDTK, fusion suicide gene involving yeast CD gene and herpes simplex virus thymidine kinase gene; shSur, Survivin-targeted small hairpin RNA; RT-qPCR, reverse transcription-quantitative polymerase chain reaction.

pYr1.1-hTERTp-yCDglyTK-shSurvivin2 is presented in Fig. 1B.

Establishment of stably transfected cell lines. hTERTp-CDTK and CDTK-shSur were administered to HCT116 cells using CPNPs. Following G418 selection, stably transfected cell lines were established.HCT116 cells transfected with hTERTp-CDTK were named HCT/CDTK, and those transfected with CDTK-shSur were named HCT/CDTK-shSur. RT-qPCR and western blot analysis were performed to determine the expression of Survivin and yCDglyTK, and immunofluorescence was conducted to determine the expression of yCDglyTK (Figs. 2 and 3, respectively). Compared with parent HCT116 cells and HCT/CDTK, mRNA and protein levels of Survivin were significantly decreased in HCT/CDTK-shSur ( $\mathrm{P}<0.01$; Fig. 2C). yCDglyTK was revealed to only be expressed in HCT/CDTK and HCT/CDTK-shSur cells (Fig. 3).

CDTK-shSur/prodrug system induced cytotoxicity. Following $48 \mathrm{~h}$ treatment with 5-FC and GCV, the OD570 of parental HCT116 cells was markedly increased compared with HCT/CDTK and HCT/CDTK-shSur cells (Fig. 4A). Over time, 


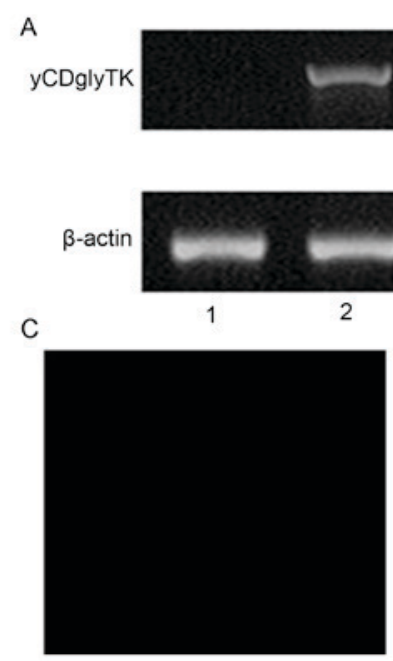

HCT116

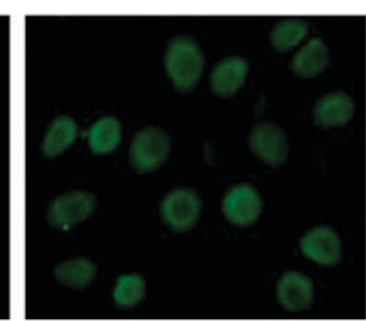

HCT/CDTK

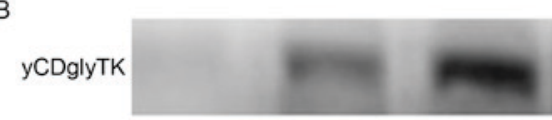

$\beta$-actin
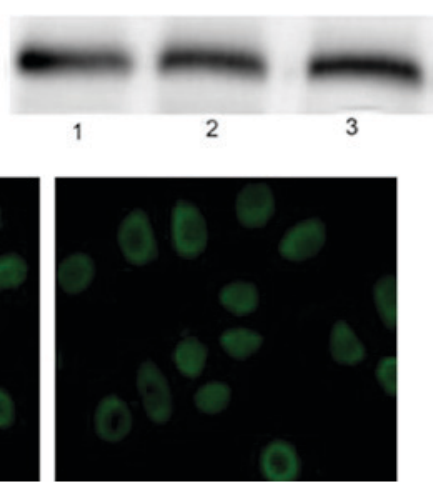

HCT/CDTK-shSur

Figure 3. Expression of yCDglyTK by transfection with CDTK-shSur. Representative yCDglyTK mRNA and protein expression were analyzed by (A) RT-qPCR and (B) western blot analysis, respectively. $\beta$-actin was used as an internal control. Lane 1, parent HCT116; lane 2, HCT/CDTK; lane 3, HCT/CDTK-shSur. (C) Representative yCDglyTK protein expression detected by immunofluorescence assays. Magnification, x200. CDTK or yCDglyTK, fusion suicide gene involving yeast CD gene and herpes simplex virus thymidine kinase gene; shSur, Survivin-targeted small hairpin RNA; RT-qPCR, reverse transcription-quantitative polymerase chain reaction.

A

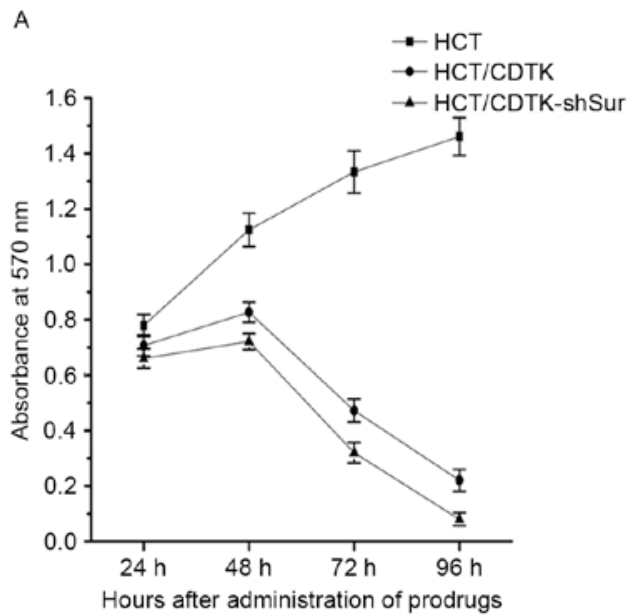

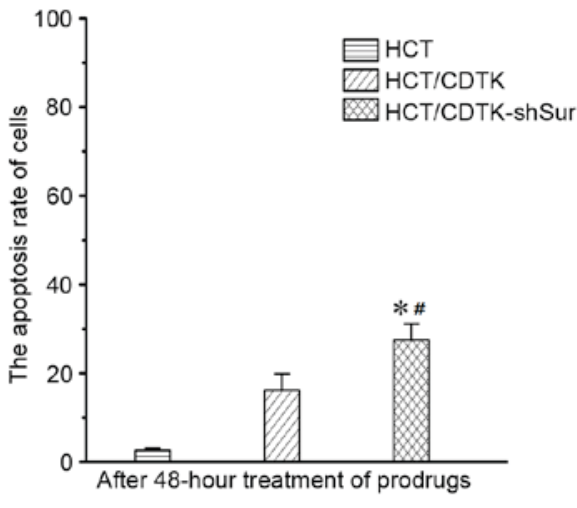

Figure 4. CTDK-shSur/prodrug system induces cytotoxicity and apoptosis. (A) Growth curves of HCT116 cells (transfected and untransfected) following administration of 5-FC and GCV. HCT116 cells (transfected and untransfected) maintained in culture medium containing 5-FC (200 $\mu \mathrm{g} / \mathrm{ml})$ and GCV $(16 \mu \mathrm{g} / \mathrm{ml})$. At 24, 48, 72 and $96 \mathrm{~h}$, cells of each group were subjected to MTT assays. Cell growth curves were plotted with culture time as the horizontal axis and OD570 as the vertical axis. (B) HCT116 cells (transfected and untransfected) were treated with prodrugs (5-FC and GCV) for $48 \mathrm{~h}$, and the apoptosis rate of each group was measured by flow cytometry. ${ }^{*} \mathrm{P}<0.05$ vs. HCT group and ${ }^{*} \mathrm{P}<0.05$ vs. HCT/CDTK group. Data are presented as mean + standard deviation. CDTK, fusion suicide gene involving yeast CD gene and herpes simplex virus thymidine kinase gene; shSur, Survivin-targeted small hairpin RNA; 5-FC, 5-fluorocytosine; GCV, ganciclovir; OD, optical density.

untransfected HCT116 cells sustained a high rate of proliferation, whereas the OD570 of HCT/CDTK and HCT/CDTK-shSur cells decreased markedly, suggesting that the majority of cells were killed. OD570 of HCT/CDTK-shSur remained the lowest throughout.

CDTK-shSur/prodrug system induced cell apoptosis. Each group was treated with prodrugs (5-FC and GCV) for $48 \mathrm{~h}$, and then subjected to flow cytometry to measure the apoptosis rate (Fig. 4B). The percentage of apoptotic cells in untransfected HCT116 cells was $2.63 \pm 0.48 \%$, in HCT/CDTK cells was $16.17 \pm 3.71 \%$ and in HCT/CDTK-shSur cells was $27.50 \pm 3.62 \%$.
The apoptosis rate of HCT/CDTK-shSur cells was significantly higher in comparison with the untransfected HCT116 and HCT/CDTK cells ( $\mathrm{P}<0.05$; Fig. 4B), indicating that the CDTK-shSur/prodrug therapy system may induce cell apoptosis more effectively.

CDTK-shSur inhibits cancer cell migration. The migration ability of HCT116 cells was measured using a wound healing assay $24 \mathrm{~h}$ following scratching. As presented in Fig. 5, compared with the parental HCT116 cells, the migration of HCT/CDTK-shSur cells decreased significantly $(\mathrm{P}<0.01$; Fig. 5B). 

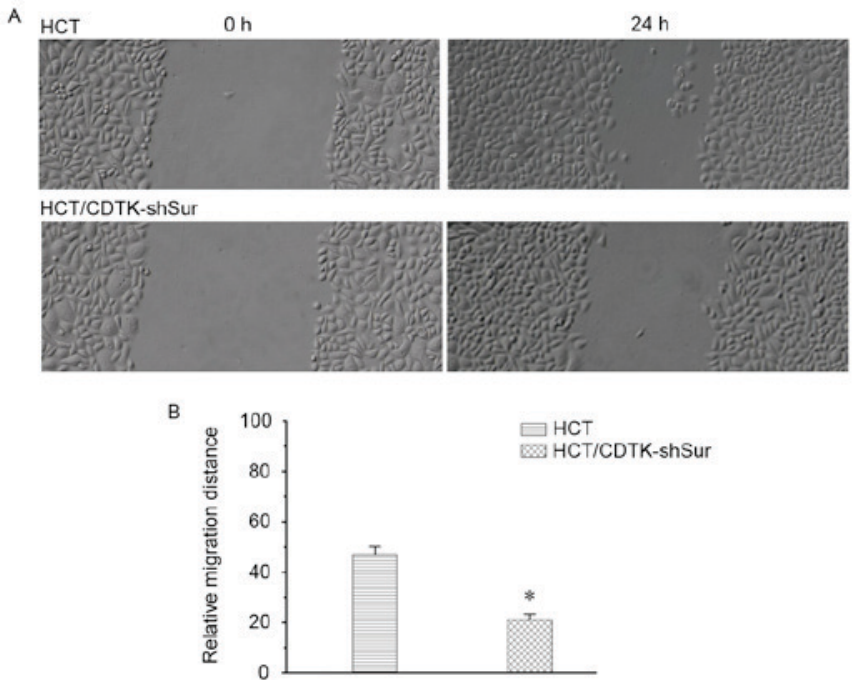

Figure 5. CDTK-shSur inhibits cancer cell migration. (A) Representative images of parent HCT116 and HCT/CDTK-shSur cells in wound healing assay (magnification, x100). (B) Quantitative analysis of the migration was made by measuring the migration distance $24 \mathrm{~h}$ after scratching. ${ }^{*} \mathrm{P}<0.01 \mathrm{vs}$. HCT. Data are presented as mean + standard deviation. CDTK, fusion suicide gene involving yeast $\mathrm{CD}$ gene and herpes simplex virus thymidine kinase gene; shSur, Survivin-targeted small hairpin RNA.

\section{Discussion}

Gene therapy has emerged as a promising strategy for treating malignant tumors (23). As the genesis, development and metastasis of cancer is a complicated process involving multiple factors (24), single gene therapy alone is not effective enough to eradicate cancer cells. Combination gene therapy may be an efficient approach to obtaining greater anti-tumor efficacy. Combination gene therapy may be achieved by co-transferring vectors carrying different genes; however, it is impossible to ensure that all of the different vectors are delivered into the cell simultaneously. The approach of one vector expressing multiple therapeutic genes has been suggested to enhance the therapeutic efficacy (25-27). In the current study, a triple-gene vector expressing Survivin-shRNA and fusion suicide gene yCDglyTK was constructed, in which Survivin-shRNA was regulated by $\mathrm{U} 6$ promoter whereas fusion suicide gene yCDglyTK was driven by hTERTp.

Different GEPTs exhibit different characteristics (7). For example, the HSV-TK/GCV system has a more powerful killing efficacy, whereas the $\mathrm{CD} / 5$-FC system exerts a superior bystander effect. Furthermore, cell type dependency may exist with GEPT, as HSV-TK/GCV is typically employed in treating gliomas (28), and the $\mathrm{CD} / 5$-FC system is often adopted in treating gastrointestinal tumors (29). Double suicide gene combined with HSV-TK/GCV and CD/5-FC may break the dependence of tumor cell types and exhibit a synergistic effect (30). The suicide gene should be expressed only in cancer cells, so GEPT may be regarded as intratumoral chemotherapy and cause little systematic toxicity. In a previous study, a vector expressing the fusion suicide gene yCDglyTK was constructed, and a CEA promoter was used to drive the expression of yCDglyTK, a treatment that specifically killed CEA-positive cancer cells (22). However, not all colorectal cancer cells are CEA-positive (31), and
yCDglyTK driven by a CEA promoter has little effect on the CEA-negative cancer cells. Therefore, in order to expand the applicability of fusion suicide gene therapy, a more prevalent promoter is required. Telomerase is activated in $>85 \%$ of all malignant tumor cells, including colorectal cancer cells, but is repressed in normal somatic cells (32-34), the transcriptional activity that is regulated by hTERTp. hTERTp was confirmed to drive specific target gene expression in various tumor cells $(9,35-37)$. Therefore, hTERTp was used in the current study to cause tumor-specific gene expression of yCDglyTK. When pYr1.1-hTERTp was delivered into both HCT116 cells and human fibroblasts, EGFP was only expressed in HCT116 cells and not in human fibroblasts, suggesting that hTERTp was specific enough to drive target gene in cancer cells.

The function of Survivin in tumor progression, metastasis and chemo-resistance has been well documented (38). In the present study, RNAi technology was used to inhibit its expression. Three Survivin-specific target sequences were selected and corresponding Survivin-shRNA expression plasmids were developed, from which the more effective one was selected. Introduction of a Survivin-targeted shRNA increased the cytotoxicity of yCDglyTK. The reasons for this synergistic effect may be as follows: Inhibition of Survivin may promote cell apoptosis and decrease cell mitosis (39); or downregulation of Survivin may maintain and enhance the sensitivity of colorectal cancer cells to cytotoxic metabolites of prodrugs. Furthermore, HCT116 cells transfected with CDTK-shSur exhibited a decreased migration ability, which determines invasiveness and metastasis of cancer cells, even without the presence of prodrugs. These data demonstrated that a combination of Survivin-siRNA and yCDglyTK may be a promising approach to treating cancer in the future.

The novel triple-gene plasmid produced in the current study may eradicate colon cancer cells and decrease their migration effectively in vitro. However, there are potential limitations of this novel system. Survivin was revealed to be expressed in normal cells, such as T-cells, hematopoietic progenitor cells, vascular endothelial cells, liver cells, gastrointestinal tract mucosa and polymorphonuclear cells (40), and participates in numerous cell processes including apoptosis, cell proliferation, cell cycle, chromosome movement, mitosis and regulation of response to cellular stress (41). The U6 promoter is not tissue-specific, and CPNPs do not target specific tissues. Strategies aiming to improve the safety of RNAi-based gene therapy are therefore required.

In conclusion, the current study has demonstrated that a combination of Survivin-targeted RNAi and suicide gene therapies exhibits a synergistic effect. Introduction of Survivin-shRNA into the CDTK/prodrug system may be an effective and feasible strategy to eradicate colon cancer cells and inhibit their migration in vitro. Although there are a number of limitations to be resolved for further application, the current study provides a novel gene therapy strategy for treating colorectal cancer.

\section{Acknowledgements}

The present study was supported by theHunan Provincial Science and Technology Program of China (grant no. 2011SK3239) and the Technology Program of Hunan Provincial Development and Reform Commission (grant no. 2011-1318). 


\section{References}

1. Ferlay J, Soerjomataram I, Dikshit R, Eser S, Mathers C, Rebelo M, Parkin DM, Forman D and Bray F: Cancer incidence and mortality worldwide: Sources, methods and major patterns in GLOBOCAN 2012. Int J Cancer 136: E359-386, 2015.

2. Marin JJ, Sanchez de Medina F, Castaño B, Bujanda L, Romero MR, Martinez-Augustin O, Moral-Avila RD and Briz O: Chemoprevention, chemotherapy and chemoresistance in colorectal cancer. Drug Metab Rev 44: 148-172, 2012.

3. Wiela-Hojeńska A, Kowalska T, Filipczyk-Cisarz E, Łapiński Ł and Nartowski K: Evaluation of the toxicity of anticancer chemotherapy in patients with colon cancer. Adv Clin Exp Med 24: 103-111, 2015.

4. Nawa A, Tanino T, Luo C, Iwaki M, Kajiyama H, Shibata K, Yamamoto E, Ino K, Nishiyama Y and Kikkawa F: Gene directed enzyme prodrug therapy for ovarian cancer: Could GDEPT become a promising treatment against ovarian cancer? Anticancer Agents Med Chem 8: 232-239, 2008

5. Hedley D, Ogilvie L and Springer C: Carboxypeptidase-G2-based gene-directed enzyme-prodrug therapy: A new weapon in the GDEPT armoury. Nat Rev Cancer 7: 870-879, 2007.

6. Karjoo Z, Chen X and Hatefi A: Progress and problems with the use of suicide genes for targeted cancer therapy. Adv Drug Deliv Rev 99: 113-128 2016.

7. Nouri FS, Wang $X$ and Hatefi A: Genetically engineered theranostic mesenchymal stem cells for the evaluation of the anticancer efficacy of enzyme/prodrug systems. J Control Release 200: 179-187, 2015.

8. Zu B, Shi Y, Xu M, You G, Huang Z, Gao M and Feng W: ARE/SUZ12 dual specifically-regulated adenoviral TK/GCV system for CML blast crisis cells. J Exp Clin Cancer Res 34: 56, 2015.

9. Rainov NG: A phase III clinical evaluation of herpes simplex virus type 1 thymidine kinase and ganciclovir gene therapy as an adjuvant to surgical resection and radiation in adults with previously untreated glioblastoma multiforme. Hum Gene Ther 11: 2389-2401, 2000.

10. Chai LP, Wang ZF, Liang WY, Chen L, Chen D, Wang AX and Zhang ZQ: In vitro and in vivo effect of 5-FC combined gene therapy with TNF-alpha and CD suicide gene on human laryngeal carcinoma cell line Hep-2. PLoS One 8: e61136, 2013.

11. Huang Q, Xia Z, You Y and Pu P: Wild Type p53 gene sensitizes rat C6 glioma cells to HSV-TK/ACV treatment in vitro and in vivo. Pathol Oncol Res 16: 509-514, 2010.

12. Park SY, Lee W, Lee J and Kim IS: Combination gene therapy using multidrug resistance (MDR1) gene shRNA and herpes simplex virus-thymidine kinase. Cancer Lett 261: 205-214, 2008.

13. Altieri DC: Survivin-The inconvenient IAP. Semin Cell Dev Biol 39: 91-96, 2015.

14. Liu JL, Gao W, Kang QM, Zhang XJ and Yang SG: Prognostic value of survivin in patients with gastric cancer: A systematic review with meta-analysis. PLoS One 8: e71930, 2013.

15. Xia H, Chen S, Huang $\mathrm{H}$ and Ma $\mathrm{H}$ : Survivin over-expression is correlated with a poor prognosis in esophageal cancer patients. Clin Chim Acta 446: 82-85, 2015.

16. Krieg A, Werner TA, Verde PE, Stoecklein NH and Knoefel WT: Prognostic and clinicopathological significance of survivin in colorectal cancer: A meta-analysis. PLoS One 8: e65338, 2013.

17. Huang J, Lyu H, Wang J and Liu B: Influence of survivin-targeted therapy on chemosensitivity in the treatment of acute myeloid leukemia. Cancer Lett 366: 160-172, 2015.

18. Jaiswal PK, Goel A and Mittal RD: Survivin: A molecular biomarker in cancer. Indian J Med Res 141: 389-397, 2015.

19. Liao Y and Tang L: Inducible RNAi system and its application in novel therapeutics. Crit Rev Biotechnol 36: 630-638, 2016.

20. Liu W, Zhu F, Jiang Y, Sun D, Yang B and Yan H: siRNA targeting survivin inhibits the growth and enhances the chemosensitivity of hepatocellular carcinoma cells. Oncol Rep 29: 1183-1188, 2013.

21. Takakura M, Kyo S, Kanaya T, Hirano H, Takeda J, Yutsudo M and Inoue M: Cloning of human telomerase catalytic subunit (hTERT) gene promoter and identification of proximal core promoter sequences essential for transcriptional activation in immortalized and cancer cells. Cancer Res 59: 551-557, 1999.
22. Liu T, Tang A, Zhang G, Chen Y, Zhang J, Peng S and Cai Z: Calcium phosphate nanoparticles as a novel nonviral vector for efficient transfection of DNA in cancer gene therapy. Cancer Biother Radiopharm 20: 141-149, 2005.

23. Libutti SK: New horizons for cancer gene therapy. Cancer Gene Ther 21: 1, 2014.

24. Backman V and Roy HK: Advances in biophotonics detection of field carcinogenesis for colon cancer risk stratification. J Cancer 4: 251-261, 2013.

25. Liu T, Ye L, He Y, Chen X, Peng J, Zhang X, Yi H, Peng F and Leng A: Combination gene therapy using VEGF-shRNA and fusion suicide gene yCDglyTK inhibits gastric carcinoma growth. Exp Mol Pathol 91: 745-752, 2011.

26. Long H, Li Q, Wang Y, Li Q, Liu T and Peng J: Effective combination gene therapy using CEACAM6-shRNA and the fusion suicide gene yCDglyTK for pancreatic carcinoma in vitro. Exp Ther Med 5: 155-161, 2013.

27. Li J, Zhang G, Liu T, Gu H, Yan L and Chen B: Construction of a novel vector expressing the fusion suicide gene yCDglyTK and hTERT-shRNA and its antitumor effects. Exp Ther Med 4: 442-448, 2012

28. Paíno T, Gangoso E, Medina JM and Tabernero A: Inhibition of ATP-sensitive potassium channels increases HSV-tk/GCV bystander effect in U373 human glioma cells by enhancing gap junctional intercellular communication. Neuropharmacology 59: 480-491, 2010

29. Zhang G, Liu T, Chen YH, Chen Y, Xu M, Peng J, Yu S, Yuan J and Zhang X: Tissue specific cytotoxicity of colon cancer cells mediated by nanoparticle-delivered suicide gene in vitro and in vivo. Clin Cancer Res 15: 201-207, 2009.

30. Niu J, Xing C, Yan C, Liu H, Cui Y, Peng H, Chen Y, Li D, Jiang $\mathrm{C}, \mathrm{Li} \mathrm{N}$ and Yang $\mathrm{H}$ : Lentivirus-mediated $\mathrm{CD} / \mathrm{TK}$ fusion gene transfection neural stem cell therapy for C6 glioblastoma. Tumour Biol 34: 3731-3741, 2013.

31. Stiksma J, Grootendorst DC and van der Linden PW: CA 19-9 as a marker in addition to CEA to monitor colorectal cancer. Clin Colorectal Cancer 13: 239-244, 2014.

32. Glybochko PV, Zezerov EG, Glukhov AI, Alyaev YG, Severin SE Polyakovsky KA, Varshavsky VA, Severin ES and Vinarov AZ: Telomerase as a tumor marker in diagnosis of prostatic intraepithelial neoplasia and prostate cancer. Prostate 74: 1043-1051, 2014.

33. Crees Z, Girard J, Rios Z, Botting GM, Harrington K, Shearrow C, Wojdyla L, Stone AL, Uppada SB, Devito JT and Puri N: Oligonucleotides and G-quadruplex stabilizers: Targeting telomeres and telomerase in cancer therapy. Curr Pharm Des 20: 6422-6437, 2014

34. Ayiomamitis GD, Notas G, Zaravinos A, Zizi-Sermpetzoglou A, Georgiadou M, Sfakianaki O and Kouroumallis E: Differences in telomerase activity between colon and rectal cancer. Can J Surg 57: 199-208, 2014.

35. Liu L, Wu W, Zhu G, Liu L, Guan G, Li X, Jin N and Chi B: Therapeutic efficacy of an hTERT promoter-driven oncolytic adenovirus that expresses apoptin in gastric carcinoma. Int J Mol Med 30: 747-754, 2012.

36. Song Y, Xin X, Zhai X, Xia Z and Shen K: Sequential combination therapy with flavopiridol and autocatalytic caspase-3 driven by amplified hTERT promoter synergistically suppresses human ovarian carcinoma growth in vitro and in mice. J Ovarian Res 7: 121,2014

37. Tian D, Sun Y, Yang Y, Lei M, Ding N and Han R: Human telomerase reverse-transcriptase promoter-controlled and herpes simplex virus thymidine kinase-armed adenoviruses for renal cell carcinoma treatment. Onco Targets Ther 6: 419-426, 2013.

38. Cheung CH, Huang CC, Tsai FY, Lee JY, Cheng SM, Chang YC, Huang YC, Chen SH and Chang JY: Survivin-biology and potential as a therapeutic target in oncology. Onco Targets Ther 6: 1453-1462, 2013

39. Li Y, Zhou Y, Zheng J, Niu C, Liu B, Wang M, Fang H and Hou C: Downregulation of survivin inhibits proliferation and migration of human gastric carcinoma cells. Int J Clin Exp Pathol 8: 1731-1736, 2015.

40. Mobahat M, Narendran A and Riabowol K: Survivin as a preferential target for cancer therapy. Int J Mol Sci 15: 2494-2516, 2014.

41. Altieri DC: Targeting survivin in cancer. Cancer Lett 332: 225-228, 2013. 